\title{
Stimulus sequence effects in concept identification
}

\author{
WILLIAM J. THOMSON \\ University of Michigan, Dearborn, Michigan 48128
}

\begin{abstract}
Previous results concerning effects of stimulus sequence variables in concept identification tasks were interpreted in terms of a variable called the logical solution sequence (LSS) length. An LSS was defined as a sequence of stimuli that contained sufficient information to enable a subject with perfect memory for the stimuli and corresponding feedback to deduce the correct solution to the problem. An experimental comparison of two LSS lengths indicated the performance was better with shorter LSSs when other sequential variables were held constant.
\end{abstract}

Previous research on concept identification has demonstrated that particular characteristics of the stimulus sequence directly affect performance. Kurtz and Hovland (1956) and Peterson (1962) found that performance improved when all possible instances of a concept were presented consecutively rather than when instances of different concepts were intermixed. Detambel and Stolurow (1956) found better performance when relevant and irrelevant dimensions were perfectly nonredundant than when irrelevant dimensions were partially redundant with the relevant dimension. Anderson and Guthrie (1966) found performance to be inversely related to the number of partially redundant irrelevant dimensions.

These results suggest an interpretation in terms of a sequential variable that may be called the logical solution sequence (LSS) length. An LSS is defined as any sequence of stimuli that contains sufficient information to enable a subject with perfect memory for the LSS (and corresponding feedback) to deduce the correct solution to the problem. More precisely, an LSS is any set of consecutive trials in which the values of the relevant dimension do not covary exactly with the values of any irrelevant dimension. For example, consider a two-cue (binary-valued) problem in which the stimulus on Trial 1 is denoted 11 . If the second stimulus is either 01 or 10 , there exists a logical solution, and the length of the LSS is two trials. However, if the second stimulus is 00 or 11 , no logical solution exists, since Cues 1 and 2 are redundant. By varying the number of trials in which only redundant information is presented, one may construct LSSs of different lengths. Since sequences in which all possible instances of a concept are presented consecutively and sequences in which relevant and irrelevant dimensions are perfectly non-

Requests for reprints should be sent to William J. Thomson, Department of Behavioral Sciences, University of MichiganDearborn, 4901 Evergreen Road, Dearborn, Michigan 48128. redundant represent shorter LSSs than their respective comparison sequences, the above results are consistent with the hypothesis that performance is inversely related to LSS length.

More direct confirmatory results were obtained by Richter (1965), who inserted particular stimuli at different locations within stimulus sequences to create LSSs of two or three trials. The probability of problem solution on a trial was found to be inversely related to the length of the LSS terminated by that trial, and the trial of last error was found to occur at a trial terminating an LSS more often than expected by chance. The present experiment was designed to test the hypothesis that LSS length is inversely related to time of problem solution when other critical variables are held constant. Two different LSS lengths were utilized, and the expected number of dimensions changing values per trial, the probability that a dimension changed values, and the placement of the LSS within the stimulus sequence were held constant.

\section{METHOD}

\section{Subjects}

The subjects were 24 female and 24 male introductory psychology students participating in partial fulfillment of a course requirement.

\section{Apparatus}

Stimuli were presented as slides that varied in color (red, blue), shape (rectangle, ellipse), size (large, small), and number of figures (one, two). Two types of slide sequences were constructed. An LSS-3 sequence consisted of overlapping LSSs of three trials, with the value of each dimension in the first stimulus randomly determined. In determining the second stimulus, the value of the single relevant dimension ( $R$ ) was changed with probability .50 and one randomly chosen irrelevant dimension $\left(R^{\prime}\right)$ was redundant with $R$. The two remaining irrelevant dimensions were nonredundant with $R$. In determining the third stimulus, the value of $R$ was changed with probability .50 and $R^{\prime}$ was nonredundant with $R$. One of the two remaining irrelevant dimensions $\left(R^{\prime \prime}\right)$ was randomly chosen to be redundant with $R$, and the other was redundant with $R^{\prime}$ (and therefore 
nonredundant with $\mathrm{R}$ ). Construction of an overlapping LSS began by considering the second stimulus as the initial stimulus and taking the new $R^{\prime}$ to be $R^{\prime \prime}$ above.

An LSS -4.5 sequence consisted of every stimulus in an LSS-3 sequence plus a derived stimulus inserted between each pair of LSS-3 stimuli. In the derived stimulus, $R$ changed values with probability .50 and both $\mathrm{R}^{\prime}$ and $\mathrm{R}^{\prime \prime}$ in the LSS were redundant with $R$. The remaining irrelevant dimension was nonredundant with R. Derived stimuli were constructed in this manner for each successive (overlapping) LSS in the LSS-3 sequence. Each stimulus from an LSS-3 sequence initiated an LSS of five trials, and each derived stimulus initiated an LSS of four trials, for an average LSS of 4.5 trials in an LSS -4.5 sequence.

Three different LSS-3 and LSS-4.5 sequences were constructed, with the constraints that no stimulus could occur twice in four consecutive trials and that no LSS could be repeated if it occurred in the five previous (overlapping) LSSs. The converse was used if a stimulus violated either of these constraints. In an LSS-3 sequence, exactly two dimensions changed values per trial, but in an LSS 4.5 sequence, either one or three dimensions changed values per trial. In both types of sequences, the probability that a dimension changed values was .50 .

\section{Procedure}

Relevant dimension, sex, and length of LSS were factors in a 4 by 2 by 2 design. Each subject was read instructions stating that the task was to learn the correct basis for classifying stimulus slides as either " $A$ " or "B." Two example slides, each the converse of the other, were shown, and the four dimensions and each of their values were pointed out to the subject. The subject was told that the solution would depend on only one of the dimensions and that the order of slide presentation was not important. The experiment was terminated when the subject had achieved 10 consecu tive correct responses.

\section{RESULTS}

The mean trial of last error was 5.13 for LSS-3 sequences and 10.58 for LSS-4.5 sequences. Analysis of variance indicated a significant main effect for LSS length $[F(1,32)=11.63, p<.01]$, with no other main effects or interactions reaching significance.

For subjects receiving LSS-4.5 sequences, the expected proportion of subjects whose criterion run began on a derived stimulus was .50 , but the observed proportion was $.75(\mathrm{z}=2.45, \mathrm{p}<.01)$. Thus, the criterion run was more likely to begin following completion of an LSS than on the last trial of an LSS. This analysis was not possible for LSS-3 sequences, since every stimulus after the second terminated an LSS.

\section{DISCUSSION}

The results clearly support the hypothesis that shorter LSSs facilitate performance in the present concept identification task. Since there were no differences between the two LSS conditions with respect to the expected number of dimensions changing values per trial, the probability that a dimension changed values, or the placement of the LSS within the sequences, the effect appears to be entirely the result of the LSS length. The finding that the trial of last error occurred on the trial terminating the LSS more often than expected by chance replicates Richter's (1965) finding and demonstrates the importance of the LSS in simple concept identification.

However, LSS should be controlled when studying more complex concept identification as well. For example, in studies that compare multidimensional concepts (e.g., conjunctive and disjunctive concepts), stimuli are typically presented in random order under various constraints. Thus, the LSS varies randomly within a subject's sequence as well as between subjects' sequences. Controlling the amount of information available to the subject at a given trial by manipulating the LSS length seems to be a desirable means of reducing error variance in these studies.

\section{REFERENCES}

Anderson, R. C., \& Guthrie, J. T. Effects of some sequential manipulations of relevant and irrelevant stimulus dimensions on concept learning. Journal of Experimental Psychology, 1966, 72, 501-504.

Detambel, M. H., \& Stolurow, L. M. Stimulus sequence and concept learning. Journal of Experimental Psychology, 1956, 51, 34-40.

Kurtz, K. H., \& Hovland, C. I. Concept learning with differing sequences of instances. Journal of Experimental Psychology, 1956, 51, 239-243.

Peterson, M. J. Some effects of the percentage of relevant cues and presentation methods on concept identification. Journal of Experimental Psychology, 1962, 64, 623-627.

Richter, M. L. Memory, choice, and stimulus sequence in human discrimination learning. Unpublished doctoral dissertation, Indiana University, 1965.

(Received for publication October 23, 1981.) 